\section{Schweizerische Gesellschaft für Intensivmedizin}

\section{Forschungsfonds}

Antragsfrist bis 30. September 2005

Die Schweizerische Gesellschaft für Intensivmedizin stellt einen neuen Fonds zur Förderung der Forschung im Bereich Intensivmedizin zur Verfügung. Die Fördersumme beträgt für zwei Jahre insgesamt 30000 Franken. Diese können einem oder, in entsprechenden Teilbeträgen (mindestens Fr. 5000.- pro Projekt), mehreren Projekten zugute kommen. Förderungswürdig sind in erster Linie Projekte mit folgenden Eigenschaften:

- klinisches oder experimentelles Projekt, das an einer schweizerischen Intensivstation durchgeführt wird;

- multizentrisches Forschungsprojekt mit anderen schweizerischen Intensivstationen;

- interdisziplinäres Forschungsprojekt;

- Förderung der öffentlichen Wahrnehmung der SGI-SSMI und der schweizerischen Intensivmedizin.

Anträge stellen können Ärztinnen und Ärzte in Weiterbildung Intensivmedizin bzw. Titelträger/innen sowie Mitglieder des Pflegepersonals einer Intensivpflegestation. Antragsteller/innen müssen ordentliches oder ausserordentliches Mitglied der Schweizerischen Gesellschaft für Intensivmedizin sein. Das Förderungsreglement und die Antragsdetails sind unter www.swiss-icu.ch verfügbar bzw. können beim Sekretariat der SGI-SSMI angefordert werden, E-Mail: sgi@imk.ch.

Anträge für die Periode 2006/2007 sind bis 30. September 2005 an das administrative Sekretariat der SGI-SSMI zu senden: Schweizerische Gesellschaft für Intensivmedizin, c/o IMK Institut für Medizin und Kommunikation AG, Münsterberg 1, 4001 Basel, E-Mail: sgi@imk.ch.

\section{Société suisse de médecine intensive}

Fonds de recherche

Délai de soumission: 30 septembre 2005

La Société suisse de médecine intensive a récemment créé un fonds destiné à soutenir la recherche dans le domaine de la médecine intensive. Il est doté d'une somme de Fr. 30 000.- par période de deux ans, qui peut être allouée à un ou plusieurs projets (minimum Fr. 5000.- par projet). Un soutien financier est accordé prioritairement à des projets de recherche qui satisfont aux critères suivants:

- projet clinique ou expérimental conduit dans une unité de médecine intensive suisse;
- caractère multicentrique de la recherche, impliquant plusieurs unités suisses de médecine intensive;

- caractère interdisciplinaire de la recherche, dans le domaine de la médecine intensive; - promotion de la visibilité de la SGI-SSMI et de la médecine intensive en Suisse.

Les requérants doivent être des médecins en formation en médecine intensive ou porteurs du titre de spécialiste ou des membres du personnel paramédical travaillant dans une unité de soins intensifs. Ils doivent être membres ordinaires ou extraordinaires de la Société suisse de médecine intensive. Le règlement du fonds de recherche et les renseignements concernant la soumission des projets sont disponibles sur le site internet www.swiss-icu.ch ou peuvent être obtenus auprès du Secrétariat de la SGI-SSMI, e-mail: sgi@imk.ch.

Les demandes de soutien pour la période 2006/2007 doivent être envoyées jusqu'au 30 septembre 2005 au Secrétariat administratif de la SGI-SSMI: Société suisse de médecine intensive, c/o IMK Institut für Medizin und Kommunikation AG, Münsterberg 1, 4001 Basel, e-mail: sgi@imk.ch.

\section{Schweizerische Arbeitsgemeinschaft für Laparoskopische und Thorako- skopische Chirurgie (SALTC)}

\section{Association Suisse pour la Chirurgie Laparoscopique et Thoracoscopique (ASCLT)}

\section{Vorstand/Comité}

Präsident/Président:

PD Dr. med. M. Schäfer, Zürich

Vizepräsident/Vice-Président:

PD Dr. med. T. Kocher, Baden

\section{Sekretär/Secrétaire:}

Dr. med. W. Gantert, Luzern

\section{Past-President:}

PD Dr L. Krähenbühl, Fribourg

Mitglieder/Membres:

Dr. med. D. Christen, Zürich; Dr. med. A. Kuhrmeier, Viganello; Prof. Dr P. Morel, Genève; PD Dr. med. M. Reymond, Magdeburg/D; Dr A. Scheiwiller, Genève; Dr M. Suter, Aigle

\section{Chargenrückruf}

\section{Supradyn, Filmtabletten,}

Zulassungsnummer 56699

Stabilitätsuntersuchungen haben gezeigt, dass die Einhaltung der Spezifikationen für den Gehalt eines Wirkstoffes (Vitamin A) über die bisher deklarierte Laufzeit von 36 Monaten nicht gewährleistet werden kann. Die Firma Bayer (Schweiz) AG ruft deshalb die Chargen des Arzneimittels mit einer Laufzeit von $36 \mathrm{Mo}$ naten aus dem Handel zurück. Packungen der betroffenen Chargen sollen bis zum 30. September 2005 auf dem Lieferweg an die jeweiligen Lieferanten retourniert werden. Die Retouren werden zu 100\% gutgeschrieben. $\mathrm{Ab}$ sofort sind Chargen mit der neuen Laufzeit von 24 Monaten erhältlich.

Betroffene Chargen

- Supradyn Filmtabletten OP 30: 2957801, 2957802, 2957803, 2959201, 2959202, 3016701, 3061101, 3061102, 3093301, 3093302;

- Supradyn Filmtabletten OP 90: 2957803, 2957804, 2959202, 2959203, 2959204, 3016702, 3016703, 3016704, 3061102, 3061103, 3061104, 3093302, 3093303 , 3093304.

Bayer (Schweiz) AG, Zürich

\section{Rappel de lot}

Supradyn, comprimés pelliculés, numéro d'enregistrement 56699

Des études de stabilité ont montré que les spécifications relatives à la teneur en un principe actif (vitamine A) ne peuvent pas être garanties pendant la durée de conservation de 36 mois déclarée à ce jour. La société Bayer (Schweiz) AG retire donc les lots du médicament avec une durée de conservation de 36 mois du marché. Les boîtes des lots concernés seront retournées, via la voie de livraison, au fournisseur correspondant avant le 30 septembre 2005. Les retours sont crédités à 100\%. Des lots avec la nouvelle durée de conservation de 24 mois sont disponibles dès maintenant.

\section{Lots concernés}

- Supradyn, 30 comprimés pelliculés: 2957801, 2957802, 2957803, 2959201, 2959202, 3016701, 3061101, 3061102, 3093301, 3093302;

- Supradyn, 90 comprimés pelliculés: 2957803, 2957804, 2959202, 2959203 , 2959204, 3016702, 3016703, 3016704, 3061102, 3061103, 3061104, 3093302, 3093303, 3093304.

Bayer (Schweiz) AG, Zürich 